\title{
Morpo-analisis ng wikangTagalog at wikang Sugbuanun'g Binisaya: Pahambing na pag-aaral
}

Bacalla, Lita $\$

Cebu Normal University, Philippines (lolita_bacalla@yahoo.com.ph)

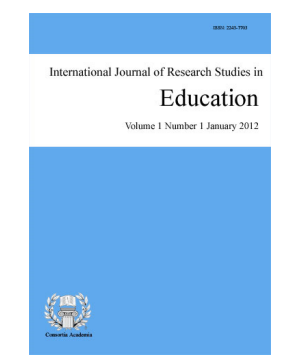

Accepted: 29 May 2019 Online ISSN: 2243-7711

OPEN ACCESS

\section{Abstrak}

Ang wika ay may kanya- kanyang kakanyahan. Layunin ng pag-aaral na matulungan ang mga Cebuano at Tagalog na maipabatid ang pagkakatulad at pagkakaiba ng mga panlapi sa Wikang Tagalog at Wikang Sinugbuanong Binisaya. 1) Masuri ang Wikang Tagalog at Wikang Sinugbuanong Binisaya sa morpemang ginagamit. 2) Matukoy ang pagkakatulad at pagkakaiba morpema ng salitang ugat. 3) Masuri ang pagkakatulad at pagkakaiba sa paglalapi at pagkakabit ng mga panlapi sa morpema. Gamit ang Kwalitatibong paraan ng pag-aaral. Deskriptibong paglalarawan ng dalawang wika. Batay sa ginagawang pagsusuri natuklasan na magkakahawig ang Wikang Tagalog at Wikang Sinugbuanong Binisaya sa mga morpemang ginagamit. Halos magkakatulad ang tuntunin sa pagkabit ng panlapi sa salitang ugat. May pagkakaiba ang dalawang wika sa termino na ginagamit. Ang morpemang makangalan at makauri ay maraming pagkakatulad samantala ang morpemang makadiwa ay magpapakaiba ng mga termino pati kung paano ito mabubuo at ang kahulugan. Hindi magkalayo ang dalawang wika kung ang pag-uuri ng morpemang salitang ugat, morpemang makangalan, morpemang makauri at morpemang makadiwa. Batay sa natuklasan, ang panlaping [a] ay panlapi na ginagamit sa wikang SB na magagamit sa tatlo sa makangalan, makauri at makadiwa na wala sa wikang Tagalog. Mas maraming panlapi ang Wikang Sinugbuanong Binisaya kaysa Wikang Tagalog ngunit kapwa magkahawatig ang dalawang wika.

Susing Salita: morpema; panlapi; salitang ugat; morpo-analisis; pahambing 


\section{Morpo-analisis ng wikangTagalog at wikang Sugbuanun'g Binisaya: Pahambing na pag-aaral}

\section{Introduksyon}

Ang wika ay may kanya- kanyang kakanyahan. Ito ay ginagamit ng tao bilang kasangkapan upang may pagkakaunawaan sa taong kausap at sa komunidad na ginagalawan. Ayon kay Arrogante (2007), ang wika ay sumasalamin sa kultura, kaisipan, kasanayan at sining ng sambayan. May sariling pekyulyaridad at sariling pamamaraan ng pag-aaral. Ipinahihiwatig na ang bawat wika ay may sariling identidad na makikita mismo kung sino ang nagsasalita at kung paano niya ginagamit sa pakikipagtalastasan. Hindi maipagkakaila na maraming mga mananaliksik na nag-aaral sa iba't ibang wika lahat ay may layunin at isa na rito ang pag-aaral ni Zorc (1987) na "The Bisayan Dialect of the Philippines subgrouping and Reconstruction" na natuklasan na kahit magkasubgrop ang mga lugar at diyalektong ginagamit may mga bahagi na hindi nagkakaunawaan. May mga bisayan ispiker na makaintindi sa ilang bisaya ngunit hindi sa lahat na pagkakataon. Tulad ng mga Cebuano ay hindi sila makakaintindi ng Waray at hindi rin sila makapagsalita ng Waray samantala ang mga Waray ay nakaunawa sa Binisayang Sebwano.

Sinusundan naman ito ng pag-aaral ng "Cebuano Verb Morphology an Application of Case Grammar" ni Lusares (1991). Sinusuri nito ang tuntunin ng pandiwa sa bawat pangungusap ng Wikang Cebuano. Natuklasan sa kanyang pag-aaral na Cebuano Verbal Morphology is rule-governed. It also has shown that semantics plays a significant part in dictating the final surface form of the verb." Nakasentro ang pag-aaral na ito sa pandiwa ngunit nakasulat ito sa Ingles at inihambing sa Ingles grammar. Kaya nahimok ang mananaliksik na magkakaroon ng hambingan pag-aaral sa wikang Tagalog at Wikang Sinugbuanong Binisaya dahil kadalasan sa ginawang pag-aaral ay nakabase sa Ingles. Isa sa mga dahilan din ay ang pagtugon sa batas R.A. 10533 na humikayat na gamitin ang unang wika sa pagtuturo mula kinder hanggang ikatlong baitang ng Mother Tongue Based Education. Hangad ng mananaliksik na makatulong ito sa kanilang lugar at bilang kagamitang pagtuturo. Bukod dito ay para mapahalagahan ang isang wika lalo na pagdating sa lokal dahil may mga maling konsepto sa pag-aaral ng wika mula sa atin kung bakit kailangan pa itong pag aralan. Halaw rin sa aklat ni Wolf (1966) "Beginning Cebuano Part I", sinabi ng may-akda na ang pagkatuto ng isang wika ay isang bagay ng nakagawiang pagbubuo. Sa ganitong dahilan kaunting panahon sa klase ang uukol sa pagpapaliwanag sa balarila ng isang wika. Ang kasing katugunan ay di lamang malilinang sa pagsasalita hinggil dito o sa pagsubok sa nilalaman nito kundi sa palagiang pagsasanay nito.

Sa aklat ni Chomsky (1957), "Synctatic Structures", nagpakita siya ng isang modelo, isang paraan ng paglalarawan ng "competence" ng isang tao sa paggamit ng wika. Katumbas ang "competence" na ito sa tinatawag ni Hamblot na "form". Sa ganitong paraan ang anyo ng wika ayon kay Chomsky ay yaong walang pagbabagong salik na nagbibigay-buhay at kahalagahan sa bawat particular na pagsasalita. Sa ibang salita, sa pamamagitan lamang kaalaman sa pinaloob na representasyon ng anyo ay maaring magkaroon ng kakayahan ang mga tao sap ag-unawa at paggamit sa isang wika. Samakatuwid kaya lang pagkakaunawaan ang dalawang taong nagtatalastasan ay sapagkat kapwa nila alam ang panloob na anyo ng wikang ginagamit. Para kay Chomsky, ang panloob na anyong ito ng isang wika ang siyang kailangang mailarawan sa balarila.

May kaugnayan ang pag-aaral na ito sa kasalukuyang pag-aaral dahil nakapokus ito sa mga pandiwa at parehong wika ang pina-aralan ngunit sa wikang Ingles ang pagkakaliwag samantala ang gagawin pag-aaral ay gamit ang wikang Filipino sa pagpapaliwag at ang lunsaran ang wikang Sinugbuanong Binisaya sa pagsusuri at batay sa pagsusuring ginawa bubuo ng glosaryo na wala sa iba. Bukod dito ay para mapahalagahan ang isang wika lalo na pagdating sa lokal dahil may mga maling konsepto sa pag-aaral ng wika mula sa atin kung bakit kailangan pa itong pag aralan. Sa Disertasyon ni Tanangkingsing (2009) natuklasan na ang panlaping Cebuano ay natatangi sa pagkakabit dahil nababago ang kahulugan kahit pareho ang anyo ng panlaping ginagamit 
samantala ang ibang lenggwahe tulad ng Bengali, Marathi (Indo-Aryan), Tibetan, Newar (TIbeto-Burman) ay manatili ang kahulugan batay sa pagkakabit nito. Mas maraming panlapi ang ikinakabit sa Sinugbuanong Binisaya kaysa ibang dayuhang lenggwahe.

Nakatuon ang pag-aaral na ito sa morpolohiyang pagsusuri ng Wikang Tagalog at Wikang Sinugbuanong Binisaya. Ang Wikang Tagalog ayon sa kasaysayan ay dating Wikang Pambansa kaya dahil sa mga linggwista ay naging maayos ang mga tuntunin at otograpiya nito. Ito ay nagsisilbing gabay sa pagbuo ng ibang wika na umiiral sa bansa. Dati naging superyor na wika ang pagtingin ng mga di Tagalog ispiker sa mga Tagalog native ispiker. Dahil sa katangian ng wika ay dinamiko at arbitraryo ay may saligang batas na nagpapatupad na ang Wikang pambansa at tawagin Filipino nangangahulugan na hindi lang Tagalog kundi kasama na ang mga wika na umiiral sa bansang Pilipinas. Hindi maiiwasan na nakabatay ang paraan sa Wikang Tagalog kung paano ang pagkakabubuo ng mga salita at morpema. Ang Wikang Sinugbuanong Binisaya naman ay isa sa mga wika sa buong Pilipinas. Ang wikang ito ay may kaugnayan sa Indonesean subgroup na galing sa Austronesean family of languages na dating tinatawag na Malayo-Polynesian family of languages na isa sa mga pinakamalaking wika sa buong mundo. Tulad ng ibang wika ang Sinugbuanong Binisaya ay mayaman din sa mga panlapi gaya ma, ting, anon, ul, on, maka, mapa at marami pang iba.

Ang pag-aaral nina Oreiro (2016) at Martelino (2016) panlapi ay malaking kaugnayan sa isasagawang pag-aaral dahil ito ay tungkol sa panlapi ginagamit sa kanilang lugar. Katutubong wika tulad ng Itawit at Maranao na kailangan pagyamanin at pagyabungin bilang ambag ng pag-aaral sa wika ng Pilipinas. Hindi man gaano ka tanyag ang wikaing ito ngunit may mga taong gumagamit nito na kailangan igalang at unawain ang wika nila. Sa mga iskolar ng wika mahalaga na kilalanin ang mga wika sa iba't ibang sulok ng bansa upang makapagsagawa ng hambingan pag-aaral sa wika. Gaya ng Wikang Sinugbuanong Binisaya ay may kakaibang paraan din sa pagkakabit ng mga panlapi sa mga salita at mas mayaman din sa mga panlapi. May pag-aaral din isinagawa sa KWF tungkol sa paglalapi ng Wikang Pampango sa pangngalan at pang-uri isiniwalat sa pag-aaral na gumamit ng unlapi, gitlapi, hulapi at kabilaan. Ito may pagbabago ng salita tulad ng asimilasyong ganap, pagkakaltas at may glottal stop deletion din sila.

Ang mga nabanggit na kaugnayan na literatura at pag-aaral ay malaking tulong para mapalawak ang kasalukuyan pag-aaral. Sinusuri sa pag-aaral na ito ang mga uri ng morpema at halimbawa nito. Alamin ang pagkakatulad at pagkakaiba ng morpemang ginagamit. Bunga nito, nilalayon ng pag-aaral na matulungan ang mga Cebuano at Tagalog na maipabatid ang pagkakatulad at pagkakaiba ng mga panlapi sa Wikang Tagalog at Wikang Sinugbuanong Binisaya.

\section{Resulta at pagtalakay}

Ang morpolohiya ay isang pag-aaral o pagsusuri sa mga morpema ng isang wika at pagsasama-sama nito upang makabuo ng isang salita. Ang morpema ay pinakamaliit na yunit ng salita na nagtataglay ng kahulugan. Ang salitang ugat (gamutpulong) ay mga salitang nagtataglay ng kahulugan kahit hindi nilalapian. Ang katumbas naman sa salitang ugat sa pinulungang Binisaya ay pulung Lintunganay o gamutpulong. Makikita sa ibaba ang mga halimbawa ng salitang ugat o gamutpulong mula sa wikang Tagalog at ang katumbas na salita sa Sinugbuanong Binisaya.

\section{Talahanyan 1}

Morpemang salitang ugat (gamutpulong)

\begin{tabular}{cc|cc}
\hline Wikang Tagalog & Wikang Sinugbuanong Bisaya & Wikang Tagalog & Wikang Sinugbuanong Bisaya \\
\hline anim & unom & hatid & hatud \\
apat & upat & itim & itom \\
bituin & bitoon & libing & lubong \\
kain & kaon & litaw & lutaw \\
hain & haon & lipad & lupad \\
halik & haluk & & \\
\hline
\end{tabular}

Tala. Ilan sa mga salita ay kadalasang ginagamit sa pang-araw-araw na gawain. 
Bacalla, L.

Ang salitang nasa kaliwang hanay ay mga halimbawa sa salitang-ugat sa Wikang Tagalog. Sa kanang hanay naman ay mga salitang-ugat o gamutpulong sa Wikang Sinugbuanong Binisaya. Napapansin na may mga salitang ugat tulad ng hatid-hatod, itim-itom, at kain-kaon na halos magkatulad talaga at sa ponema lang ang nabago. Ang Tagalog ay may ponemang/i/ at ang binisaya ay may ponemang/o/. Mula ponemang /i/ ay nabago ito sa binisaya ng ponemang /o/. Ang hatid-hatod, itim-itom, at kain-kaon magkakatulad ng kahulugan. Kaya masasabing magkalapit sa isa't isa ang dalawang wika kaya hindi ito mahirap pag-aralan ng mga di-native ispeker. May pagkakataon sa wikang Tagalog ay ponema[i] ay nagiging ponemang [o] sa Sinugbuanong Binisaya.

Ayon kay Lachica (1994) dapat tandaan na ang wika ay hindi lamang puro tunog at pagbigkas. Mayroon din itong regulasyon sa palabigkasan, gramatika at semantika. Nasusunod ang mga ito sa pang-araw-araw na pakikisalamuha at pakikipagtalastasan ng tao sa kanyang komunidad. Isinasaad dito na ang wika ay hindi lamang nakatuon sa pagbigkas sapagkat ang bawat wika ay maaaring mahalaw sa pakikipaghalubilo sa bawat isa o di-kaya'y sa pook na kinatayuan kung saan puspusang naisakatuparan dito ang ebolusyon at pagkakaiba ng wika tulad ng wikang Tagalog at wikang Sinugbuanong Binisaya.

\section{Talahanayan 1.1}

Morpemang salitang-ugat

\begin{tabular}{cc|cc}
\hline Wikang Tagalog & Wikang Sinugbuanong Binisaya & Wikang Tagalog & Wikang Sinugbuanong Binisaya \\
\hline dayo & day-o & pangos & pang-os \\
hagot & hag-ot & pasan & pas-an \\
hilamos & hilam-os & puson & pus-on \\
kanin & kan-on & sabit & sab-it \\
kawit & kaw-it & sipon & sip-on \\
kaysa & kay-sa & tabang & tam-ang \\
lagok & lag-ok & tamis & \\
luya & luy-a & & \\
\hline
\end{tabular}

May mga salitang magkapareho ang baybay at kahulugan ang Wikang Tagalog at Wikang Sinugbuanong Binisaya ngunit ang mga salitang ito magkaiba ang pagbigkas dahil ang salitang Sinugbuanong Binisaya ay nagkakaroon ng impit na tunog sa pagitan ng patinig at katinig. Halimbawa ang mga salitang nasa itaas tulad ng salitang bago ay naging bag-o. Ang salitang tagalog ay bago na nangangahulugan bago ay walang gitling samantala ang Sinugbuanong Binisaya ay may gitling ngunit magkatutulad na magkatulad ang kahulugan. Luya ay naging luy-a sa Sinugbuanong Binisaya ganoon din ang padron sa mga salitang makikita sa talahanayan.Malumanay ang pagbigkas ng mga salitang Tagalog at ang wikang Sinugbuanong Binisaya. Ang tuntunin ng wikang Sinugbuanong Binisaya ay gumamit ng giyon o gitling sa paghihiwalay ng patinig o patingog at katinig o katingog.

\section{Talahanayan 1.2}

Morpemang salitang ugat

\begin{tabular}{cc}
\hline Wikang Tagalog & Wikang Sinugbuanong Binisaya \\
\hline lamang & lang \\
salamin & samin \\
mababaw & babaw \\
salapi & sapi \\
bubuyog & buyog \\
butiki & tiki \\
bulaklak & bulak \\
daan & dan \\
dalaga & daga \\
\hline
\end{tabular}

Sa talahanayan sa itaas ay makikita ang mga salitang magkatulad ang kahulugan ngunit may pagkakaltas na nangyayari. Ang wikang Tagalog ay pormal ang mga salita samantala ang Wikang Sinugbuanong Binisaya ay nagkakaltas. Halimbawa ang salitang butiki ay naging tiki, bubuyog ay buyog, lamang ay lang, mababaw ay mabaw mataas ay taas, malayo ay layo, dalaga ay daga, kanila ay ila, kaniya ay iya. Ang salitang ito ay may 
pagkakaltas na nangyayari sa unahan pantig. Ang kasunod na mga salitang may pagkakaltas ang salitang bulaklak ay naging bulak, daan ay dan, salapi ay sapi, salamin ay samin, bahay ay bay, dalaga ay daga. Kung pagtuunang pansin ang mga salita ito ay may pagkakaltas sa gitnang patinig mula wikang Tagalog. At ang ilang salitang tulad ng ulo ay naging ǔ, ang opo ay o na kung saan pagkakaltas sa hulihang pantig ng salita. Ang wikang Sinugbuanong Binisaya ay di-pormal at kolokyal ang mga salita kaya madalas mapagkamalang walang mudo o walang galang bukod sa hindi pagsasalita ng opo at po ang mga salita din nila ay kinakaltasan. Ang Wikang Tagalog ay ang mga salitang pormal kung pagbabatayan. Isa din sa mga kultura ng mga Sebuano ay ay magpakatamad kung magsalita at palaging nagmamadali at laging tapusin agad ang pagsasalita. Bunga nito ay nagkakaroon ng mga maling persepsyon ng iba sa tao dahil sa paggamit ng wika at salita sa pakikipagtalastasan ngunit nagkakaunawaan dahil may pagkakatulad at may konting pagkakaiba.

May mga salitang sa wikang Tagalog at wikang Sinubuanong Binisaya ay magkakatulad ng kahulugan ngunit may pagkakaiba tulad ng mga ponema, may pagkakaltas at pagpapantig.

\subsection{Morpemang Makangalan}

Ang sinusuri ay batay sa morpema at ang kahulugan nito kung paano ginagamit. Ang panlapi ay mga kataga na walang saysay o kahulugan kapag hindi ikinakabit sa salitang-ugat ngunit kapag ikinakabit na ito ay makapagbago rin ng kahulugan sa mga salitang-ugat na maaring mauuri sa morpemang makangalan makangan, makauri (makapungway) at makadiwa (makapunglihuk). Ang Sinugbuanong Binisaya ay may panlapi din na tinatawag na linanggikitan. Ang unlapi ay tinatawag itong unanggikit. Ang gitlapi naman ay tinatawag na tunganggikit at ang hulapi naman ay tinatawag itong pangulhinggikit. Pungngan ang tawag ng pangngalan sa pinulungan Binisaya.

Ipinapakita sa talahanayan 2 ang paghahanay ng mga salita nasa morpemang makangalan o makangan.

\section{Talahanayan 2}

Morpemang Makangalan -Makangan

\begin{tabular}{|c|c|c|c|}
\hline Wikang Tagalog & Kahulugan & Wikang Sinugbuanong Binisaya & Kahulugan \\
\hline makangalan & \multicolumn{3}{|c|}{ makangan } \\
\hline -an & \multirow[t]{16}{*}{$\begin{array}{l}\text { nangangahulugan ito ng pook na } \\
\text { kinaroonan o ginagampanan o panahon }\end{array}$} & ka- an/anan & $\begin{array}{l}\text { nangangahulugan ito ng pook na } \\
\text { kinaroonan o ginagampanan }\end{array}$ \\
\hline asinan & & kasabutan & \\
\hline usapan & & kasagingan & \\
\hline taguan & & kadaruhan & \\
\hline pasukan & & kahumayan & \\
\hline aklatan & & kakahuyan & \\
\hline bigasan & & agianan & \\
\hline palayan & & bakasyunanan & \\
\hline & & tagboanan & \\
\hline & & lusotanan & \\
\hline & & uliananan & \\
\hline & & bughaanan & \\
\hline & & kantahanan & \\
\hline & & palitanan & \\
\hline & & abotanan & \\
\hline & & sibyaanan & \\
\hline ka-an & \multirow[t]{5}{*}{$\begin{array}{l}\text { nangangahulugan ng kaisipang } \\
\text { abstrakto, pook }\end{array}$} & ka-an & $\begin{array}{l}\text { nangangahulugan ng kaisipang } \\
\text { abstrakto, pook }\end{array}$ \\
\hline kapatiran & & kawanangan & \\
\hline kagandahan & & kahimayaan & \\
\hline karagatan & & kalibutan & \\
\hline kaligayahan & & & \\
\hline tag- & \multirow[t]{9}{*}{ nagsasaad ng panahon } & ting- & nagsasaad ng panahon \\
\hline halimbawa: & & tingtugnaw & \\
\hline tagtuyot & & ting uwan & \\
\hline tag init & & tingbagyo & \\
\hline taglamig & & ting-init & \\
\hline taglagas & & tinghuwaw & \\
\hline tagsibol & & ting-ani & \\
\hline tag-araw & & ting- itlog & \\
\hline tag-ulan & & ting-anak & \\
\hline
\end{tabular}


Bacalla, L.

Talahanayan 2 ... pagpapatuloy

\begin{tabular}{|c|c|c|c|}
\hline Wikang Tagalog & Kahulugan & Wikang Sinugbuanong Binisaya & Kahulugan \\
\hline taga- & $\begin{array}{l}\text { nagsasaad ng lahing pinagmulan o } \\
\text { gawain }\end{array}$ & tag-/tig- & $\begin{array}{l}\text { nagsasaad pagmamay ari at } \\
\text { nagsasaad ng lahing pinagmulan ng } \\
\text { gawain }\end{array}$ \\
\hline tagacavite & & tag-iya (may-ari) & \\
\hline tagaradyo & & tagdumala(tagapamahala) & \\
\hline taga-alok & & tagtungod( & \\
\hline tagapanayam & & tigkalus(taga-igib) & \\
\hline tagabigay & & tighatod(tagahatid) & \\
\hline & & tighukad(tagasugkay) & \\
\hline ka- & nangangahulugan itong relasyon & ka-, ka-an, ka-han & nangangahulugan itong relasyon \\
\hline kapatid & & kabanayan & \\
\hline kabaro & & kapulongan & \\
\hline kapamilya & & kaigsoonan & \\
\hline kapuso & & kaig -agawan & \\
\hline kanayon & & kasilinganan & \\
\hline kalaro & & kahigalaan & \\
\hline kasama & & kabaranggayan & \\
\hline katuwang & & katigulangan & \\
\hline katulong & & kaparyentehan & \\
\hline kaagapay & & & \\
\hline $\begin{array}{l}\text { mag-+pag-uulit ng } \\
\text { unang pantig ng } \\
\text { salitang-ugat }\end{array}$ & $\begin{array}{l}\text { nangngahulugan ng gawain o } \\
\text { hanapbuhay }\end{array}$ & mag+ paguulit ng unang pantig & nagsasaad ng gawain o hanapbuhay \\
\hline magtataho & & magtutudlo(guro) & \\
\hline magtatanim & & magsusulat(writer) & \\
\hline mag-aaral & & magtutuon & \\
\hline maglalaba & & magpupulong & \\
\hline & & maghuhukom & \\
\hline & & mag-uuma & \\
\hline & & magdadaro & \\
\hline & & magsisibya & \\
\hline $\begin{array}{l}\text { mang-+unang } \\
\text { salita }\end{array}$ & $\begin{array}{l}\text { nangangahulugan ng gawain o } \\
\text { hanapbuhay }\end{array}$ & ma-+ pag uulit ng unang & $\begin{array}{l}\text { nangangahulugan ng gawain o } \\
\text { hanapbuhay }\end{array}$ \\
\hline mang-aawit & & manganganta & \\
\hline mangingisda & & mananagat & \\
\hline manggagamot & & mananambal & \\
\hline mapag- & nagsasasaad na may ugali & mapa- & nagsasasaad na may ugali \\
\hline mapagtimpi & & mapahitas on & \\
\hline mapagkumbaba & & mapaubsanon & \\
\hline mapaghiganti & & mapailubon & \\
\hline mapagbiro & & mapahiyumon & \\
\hline mapagbintang & & mapanganduyon & \\
\hline mapaghinala & & mapasagarun & \\
\hline & & $-\mathrm{a}$ & $\begin{array}{l}\text { ginagamit ang panlaping [a] pagbibigay } \\
\text { diin sa pangalan }\end{array}$ \\
\hline & & tuig-taon - tuiga & \\
\hline & & bulak-bulaklak- bulaka & \\
\hline & & baboy-baboy-baboya & \\
\hline
\end{tabular}

\subsection{Morpemang Makauri}

Ang morpemang Makauri ay ang mga panlaping ikinakabit sa salitang-ugat upang makabuo ng mga pang-uri. Ang tawag sa pang-uri sa Sinugbuanong Binisaya ay makapungway galing sa salitang pulong na naghulagway na ang ibig sabihin salitang naglalarawan.

Binibigyang tuon sa talahanyan 3 ang mga halimbawa ng mga morpemang makauri. 
Morpo-analisis ng wikangTagalog at wikang Sugbuanun'g Binisaya: Pahambing na pag-aaral

Talahanayan 3

Morpemang Makauri (Makapungway)

\begin{tabular}{|c|c|c|c|}
\hline Wikang Tagalog & Kahulugan & Wikang Sinugbuanong Binisaya & Kahulugan \\
\hline makauri & & makapungway & \\
\hline ma- & $\begin{array}{l}\text { nagsasaad ng pagkakaroon na isinasaad } \\
\text { ng salitang-ugat o pagiging marami }\end{array}$ & ka- & $\begin{array}{l}\text { nagtataglay o naglalarawan sa } \\
\text { nararamdaman }\end{array}$ \\
\hline mayaman & & kaguol & \\
\hline mabuti & & kamingaw & \\
\hline makisig & & kahadluk & \\
\hline mapuno & & kapungot & \\
\hline makitid & & kalagot & \\
\hline maganda & & kakapoy & \\
\hline mayabang & & kagutom & \\
\hline mala & & on/hon & $\begin{array}{l}\text { ito ay nagsasaad ng pagkakaroon ng } \\
\text { isinasaad ng salitang-ugat upang } \\
\text { maipakita ang katangiang higit pa } \\
\text { sa karaniwang dami, laki, tindi at } \\
\text { iba pa. }\end{array}$ \\
\hline malasibuyas & & humukon & \\
\hline malapalasyo & & gahion & \\
\hline malarosas & & hilason & \\
\hline malakabayo & & taason & \\
\hline malaporcelana & & gamayon & \\
\hline pala & $\begin{array}{l}\text { nagsasaad ito ng katangiang palagi o } \\
\text { pauli- ulit ginagawa }\end{array}$ & pala & $\begin{array}{l}\text { nagsasaad ito ng katangiang palagi } \\
\text { o paulit-ulit ginagawa }\end{array}$ \\
\hline palasulat & & palauyab & \\
\hline palabiro & & palahubog & \\
\hline palangiti & & palainom & \\
\hline palaisip & & palakaon & \\
\hline palakain & & palailis & \\
\hline ma-in-hin & $\begin{array}{l}\text { nangangahulugan ito ng pagtataglay ng } \\
\text { mataas na antas ng isinasaad ng salitang } \\
\text { ugat }\end{array}$ & $\begin{array}{l}\text { ma-in- } \\
\text { on }\end{array}$ & $\begin{array}{l}\text { nangangahulugan ito ng pagtataglay } \\
\text { ngmataas na antas ng isinasaad ng } \\
\text { salitang ugat } \\
\text { nangangahulugan ito ng positibong } \\
\text { katangian ng tao }\end{array}$ \\
\hline \multirow{10}{*}{$\begin{array}{l}\text { madasalin } \\
\text { maramdamin } \\
\text { matulungin } \\
\text { masunurin } \\
\text { maawain }\end{array}$} & & matinabangon & \\
\hline & & masinabtanon & \\
\hline & & matinud anon & \\
\hline & & mabinantayon & \\
\hline & & $\mathrm{a}$ & $\begin{array}{l}\text { ginagamit sa pagbibigay diin sa } \\
\text { pang-uri }\end{array}$ \\
\hline & & bugnaw- malamig bugnawa & \\
\hline & & lami-masarap-lamia & \\
\hline & & daghan-marami-daghana & \\
\hline & & gamay-maliit-gamaya & \\
\hline & & dako-malaki-dakoa & \\
\hline
\end{tabular}

Kapansin-pansin sa talahanayan na ang Wikang Tagalog (WT) ay may panlaping ma, mala, pala, ma-in-hin at sa Wikang Sinugbuanong Binisaya (WSB) naman ay may ka, on, hon, pala, ma-in-on. Walang panglaping ma na nag-iisa sa WSB hindi tulad sa WT may panlapi ma ang WSB ngunit may katimbang ito ng mga panlaping in, on, at hon. Sa panlaping ka(unlapi) naman sa WSB ngunit wala naman sa WT. Tumpak ang panlaping pala ay parehong-pareho talaga sa baybay at sa kahulugan kapag ito ay ikinakabit sa mga salitang ugat na nangangahulugan paulit-ulit na ginagawa. Ang dalawang wika ay may panlapi din na in ngunit magkaiba ito sa posisyon sa pagkabit. Sa talahanayan ipinapakita nito na ang in sa WT ay nasa hulihan o ginagamit bilang hulapi samantala sa SWB ay kinakabit bilang gitlapi ngunit ang pagsasamasama ng mga panlapi ito ay magkatulad na magkatulad ang kahulugan.

Sa kabuuan ang mga panlapi na ginagamit sa dalawang wika ay magkatulad na magkatulad ang tuntunin at ang pagkabuo sa morpoemang makauri.

\subsection{Morpemang Makadiwa (makalihuk)}

Ang Morpemang Makadiwa ay ang mga panlapi ng ginagamit sa pagbuo ng mga pandiwa. Ang katumbas ng pandiwa sa binisaya ay punglihuk na galing sa salitang pulong ug lihuk na nangangahulugan ng mga salitang 
Bacalla, L.

nagsasaad ng kilos o aksyon. Sa tulong ng mga panlaping ito makakabuo ito ng mga salitang nag-aanyong pandiwa kapag kinakabitang ng mga panlaping ito.

Makikita sa talahanayan 4 ang mga halimbawang morpemang makadiwa.

\section{Talahanayan 4}

Morpemang Makadiwa-Makapunglihuk

\begin{tabular}{|c|c|c|c|}
\hline Wikang Tagalog & Kahulugan & Wikang Sinugbuanong Binisaya & Kahulugan \\
\hline makadiwa & & makalihuk & \\
\hline i- & $\begin{array}{l}\text { nagsasaad ng paggamit ng isang bagay } \\
\text { at nasa pokus kagamitan }\end{array}$ & i-/ipa- & $\begin{array}{l}\text { nagsasaad na gagawin pa ang kilos } \\
\text { nagsasaad din ng paggamit ng isang } \\
\text { bagay }\end{array}$ \\
\hline ibigay & & ihatag & \\
\hline isulat & & itunol & \\
\hline ibasa & & ililong & \\
\hline ihiwalay & & ilabay & \\
\hline iguhit & & ihilak & \\
\hline iinom & & ikatawa & \\
\hline um- & $\begin{array}{l}\text { ang panlaping um ay maaaring unlapi o } \\
\text { gitlapi ayon kung ano ang unang } \\
\text { ponema ng salitang-ugat na nilalapian. } \\
\text { ito'y ginagamit sa pokus tagaganap ng } \\
\text { kilos }\end{array}$ & gi & $\begin{array}{l}\text { nagsasaad na kasaluyang } \\
\text { isinasagawa ang kilos na walang } \\
\text { pag-aalinglangan }\end{array}$ \\
\hline tumakbo & & gilabay & \\
\hline lumundag & & gitabunan & \\
\hline lumayas & & gihatag & \\
\hline uminom & & gitaho & \\
\hline lumiban & & gilabay & \\
\hline ma- & $\begin{array}{l}\text { nagsasaad ng kakayahang gawin ang } \\
\text { kilos na isinasaad ng salitang ugat at } \\
\text { nagsasaad ng di-sinasadyang pagganap }\end{array}$ & mi- & $\begin{array}{l}\text { nagsaad na kasalukuyan ginagawa } \\
\text { at nasa pokus tagaganap }\end{array}$ \\
\hline masulat & & midawat & \\
\hline malaro & & mikaon & \\
\hline maluto & & milabay & \\
\hline maupo & & mikatawa & \\
\hline mag- & $\begin{array}{l}\text { ito'y laging inilagay sa unahan ng } \\
\text { salitang-ugat. ginagamit din sa pokus } \\
\text { tagaganap at nagsasaad ng kilos. ito'y } \\
\text { likas na pandiwang palipat }\end{array}$ & mo & $\begin{array}{l}\text { nagsasaad ng kilos na } \\
\text { panghinaharap o akmang gagawin } \\
\text { ang isang bagay o tagaganap ng } \\
\text { kilos }\end{array}$ \\
\hline magsulat & & moduaw & \\
\hline maglaro & & mokalawat & \\
\hline mag-araro & & motabang & \\
\hline maghakot & & mosiyagit & \\
\hline magbasa & & molakaw & \\
\hline magsayaw & & moamen & \\
\hline ka-an/han & $\begin{array}{l}\text { may kahulugang gawin sa paksa ang } \\
\text { kilos ng pandiwa }\end{array}$ & nag & $\begin{array}{l}\text { nagsasad sa kilos ng kasalukyang } \\
\text { ginagawa }\end{array}$ \\
\hline kasuyaan & & nagyawyaw & \\
\hline kabaliwan & & naghilak & \\
\hline kabagutan & & naglaba & \\
\hline kainisan & & nagbakho & \\
\hline kagalitan & & nagdagan & \\
\hline isa- & $\begin{array}{l}\text { may kahulugan ilagay sa kalagayan nasa } \\
\text { salitang-ugat ang paksa }\end{array}$ & nang- & $\begin{array}{l}\text { nagsasaad ng kilos na akmang } \\
\text { ginagawa }\end{array}$ \\
\hline isatabi & & nanghilam-os & \\
\hline isakatawan & & nangadye & \\
\hline isakwento & & nanglimpy0 & \\
\hline isapelikula & & nanglaba & \\
\hline isabuhay & & nanghimasa & \\
\hline maki- & $\begin{array}{l}\text { nagsasaad ng pakiusap upang sumama } \\
\text { sa ibang tao sa pagganap sa kilos ng } \\
\text { pandiwa }\end{array}$ & mag- & $\begin{array}{l}\text { ito ay laging inilalagay sa unahan } \\
\text { ng salitang-ugat. ginagamit din sa } \\
\text { pokus na tagaganap at nagsasad ng } \\
\text { kilos. ito'y likas sa pandiwang } \\
\text { palipat }\end{array}$ \\
\hline makibigay & & maghulat & \\
\hline makisuyo & & magtuon & \\
\hline makilagay & & magsulat & \\
\hline makihulog & & magpasilong & \\
\hline makihulog & & maghimo & \\
\hline
\end{tabular}


Morpo-analisis ng wikangTagalog at wikang Sugbuanun'g Binisaya: Pahambing na pag-aaral

Talahanayan 4 ...pagpapatuloy

\begin{tabular}{|c|c|c|c|}
\hline Wikang Tagalog & Kahulugan & Wikang Sinugbuanong Binisaya & Kahulugan \\
\hline $\begin{array}{l}\text { mang-(mam-, } \\
\text { man-) }\end{array}$ & $\begin{array}{l}\text { may kahulugang maraming pagganap, } \\
\text { kasing kahulugan at katulad ng mga- sa } \\
\text { pokus }\end{array}$ & maka- & nagsasaad ng kakayahan sa pagkilos \\
\hline mambato & & makalupad & \\
\hline manghimok & & makatugpa & \\
\hline mambutas & & makadungog & \\
\hline manghabol & & makakita & \\
\hline manghalik & & makalukso & \\
\hline magka- & $\begin{array}{l}\text { nagsasaad ng pagkakaroon ng isang } \\
\text { bagay }\end{array}$ & maka- & $\begin{array}{l}\text { nagsasaad ng pagkakaroon ng isang } \\
\text { bagay }\end{array}$ \\
\hline magkasalapi & & makasapi & \\
\hline magkalupa & & makauyab & \\
\hline magkabahay & & makabana & \\
\hline magkaroon & & makayuta & \\
\hline magkaganito & & makasakyanan & \\
\hline maka- & $\begin{array}{l}\text { nagsasaad ng kakayahang gawin ang } \\
\text { kilos na nasa salitang ugat }\end{array}$ & maga- & $\begin{array}{l}\text { nagsasaad ng kakayahang gawin } \\
\text { ang kilos na nasa salitang ugat }\end{array}$ \\
\hline makabasa & & magahatod & \\
\hline makalaro & & magahigda & \\
\hline makaguhit & & magahilak & \\
\hline makalihok & & magapamatuod & \\
\hline makagamot & & magalutos & \\
\hline makabigay & & magapamat & \\
\hline magsi- & $\begin{array}{l}\text { nasa anyong maramihan at nasa pokus } \\
\text { tagaganap }\end{array}$ & makig- & $\begin{array}{l}\text { nagsasaad ng kilos na ginanap na } \\
\text { may kasama }\end{array}$ \\
\hline magsilaro & & makig-istorya & \\
\hline magsisulat & & makig-uban & \\
\hline magsilaba & & makighinabi & \\
\hline magsitulong & & makigsayaw & \\
\hline magsiluto & & makigsuroy & \\
\hline mag,-an/-han & $\begin{array}{l}\text { nagsasaad ito ng tambingang kilos ng } \\
\text { sabayan }\end{array}$ & mag-in,-ay & $\begin{array}{l}\text { nagsasaad ito ng tambingang kilos } \\
\text { ng sabayan }\end{array}$ \\
\hline maghiyawan & & magpinatiray & \\
\hline magtakbuhan & & magkinulatahay & \\
\hline magmurahan & & magnilibakay & \\
\hline magsuyuan & & magsinabtanay & \\
\hline magkainan & & magtinabangay & \\
\hline magsubuan & & magbinatiay & \\
\hline papag-in/hin & $\begin{array}{l}\text { nagsasaad na payagan o utusan ang } \\
\text { tumutukoy sa paksa na gawin ang kilos } \\
\text { na isinasaad ng salitang ugat }\end{array}$ & pag- & nagsasaad ng pagsasagawa ng kilos \\
\hline papaglakbayin & & paghubit & \\
\hline papagsulatin & & pagsugyot & \\
\hline papaggupitin & & paghukom & \\
\hline papagbasahin & & pag-uyon & \\
\hline \multirow[t]{6}{*}{ papagwalisin } & & pagsalmut & \\
\hline & & $-\mathrm{a}$ & $\begin{array}{l}\text { ginagamit ang panlaping ito sa } \\
\text { pagbago mula pang-uri tungo sa } \\
\text { pandiwa na nagsasaad ng pag-uutos }\end{array}$ \\
\hline & & sagpa-sampal & \\
\hline & & sagpaa-sampalin mo & \\
\hline & & sumbag-suntok & \\
\hline & & sambaga-suntukin mo & \\
\hline
\end{tabular}

Sa Wikang Tagalog may mga panlapin /i/, /um/, /ma/, /mag/ /ka-an/, /han//isa//maka//magsi//mag//an-han//papag//in-hin/. Sa Wikang Sinugbuanong Binisaya naman ay may /i//ipa//gi//mi//mo//nag//mag//maka//maga//makig//pag//mag//in-ay/.

Walang panlaping um, hin, papag sa SWB sa WT naman ay walang din gi, mo, nang, makig, mi at mag. Maraming hindi magkakatulad na panlapi na ginagamit sa dalawang wika ngunit kung papansinin mabuti na may kanya-kanya itong gamit upang makabuo ng morpemang makadiwa. Ang panlaping tulad ng i at ipa ay magakakahawig ang gamit nito sa dalawang wika pati narin sa panlaping magka at maka. Ang mag at mo ay halos magkakatulad din sa tuntuni at anyo ng paggamit. 


\section{Natuklasan}

Batay sa ginagawang pagsusuri natuklasan na magkakahawig ang Wikang Tagalog at Wikang Sinugbuanong Binisaya sa mga morpemang ginagamit. Halos magkakatulad ang tuntunin sa pagkabit ng panlapi sa salitang ugat. May pagkakaiba ang dalawang wika sa termino na ginagamit. Ang morpemang makangalan at makauri ay maraming pagkakatulad samantala ang morpemang makadiwa ay magpapakaiba ng mga termino pati kung paano ito mabubuo at ang kahulugan. May mga panlapi na wala sa dalawang wika na kakaiba talaga.

Ang dalawang wika na Wikang Tagalog at Wikang Sinugbuanong Binisaya ay may mga makahawig na mga morpema ng salitang ugat na pareho ang kahulugan ngunit nagkaiba sa ponemang ginagamit. Ang tagalog ay kadalasan gumamit ng ponemang [i], samantala ang Wikang Sinugbuanong Binisaya ay ponemang [o], at [u] ang ginamit.

Ang wikang Tagalog ay pormal ang mga salita at mahinahon kung magsalita samantala ang wikang Sinugbuanong Binisaya ay madalas magkaltas dahil sa kultura nitong maypakatamad kung magsalita at palaging nagamamadali at gusto agad tapusin ang pagsasalita.

1. Ang wikang Tagalog ay malumay ang mga salita samantala ang wikang Sinugbuanong Binisaya nagkakaroon ng impit ng mga salita sa paggamit ng gitling o giyun.

2. Halos magkakatulad ang pagkakabit at paglalapi sa morpemang makangalan, nagkaiba ito sa terminong ginagamit, ngunit mas maraming panlapi ang Sinugbuanong Binisaya kaysa sa Wikang Tagalog.

3. Nagkakaiba ang paraan ng pagkakabit at paglalapi sa morpemang makadiwa o makalihuk. Maraming panlapi ang ginagamit, may panlaping wala ang Wikang Tagalog tulad ng [a], [i], [hi],[ig],[gi],[ul], [hay], [ming] ang panlaping [i], [hi],[ig],[gi] ay ginagamit bilang panlaping unahan at hulihan sa Wikang SB, samantala sa Wikang Tagalog ay kadalasan sa unlapi , at gitlapi, at walang panlaping [a].

4. Ang panlaping [a] sa wikang Sinugbuanong Binisaya ay ginagamit sa tatlong morpemang makangalan, makauri, at makadiwa at ang panlaping ito ay wala sa wikang Tagalog.

\section{Kongklusyon}

Hindi magkalayo ang dalawang wika kung ang pag-uuri ng morpemang salitang ugat, morpemang makangalan, morpemang makauri at morpemang makadiwa. Batay sa natuklasan, ang panlaping [a] ay panlapi na ginagamit sa wikang SB na magagamit sa tatlo sa makangalan, makauri at makadiwa na wala sa wikang Tagalog. Mas maraming panlapi ang Wikang Sinugbuanong Binisaya kaysa Wikang Tagalog ngunit kapwa magkahawatig ang dalawang wika. Hindi mahirap pag-aralan ang dalawang wika.

\subsection{Rekomendasyon}

Batay sa mga resulta ng pagsusuri, nabuo ang sumusunod na rekomendasyon:

$>\quad$ Magkaroon pa ng ibayong pag-aaral ng ilang bahagi ng pananalita para sa istruktura ng Wika lalo na sa Wikang Sinugbuanong Binisaya.

$>\quad$ Magkaroon ng ibayong pag-aaral na may kinalaman sa Ponolohiya ng Sinugbuanong Binisaya at ihambing ito sa Wikang Tagalog.

$>\quad$ Sanayin ang mga mga estudyante na magkaroon ng hambingang pagsusuri ng mga wika sa bansa. 


\section{Sanggunian}

Calara, L. P. (1996). Mga panlaping makadiwa na gamit ng mga nagsasalit ng barayti ng Filipino sa mga pakikipag-ugnayang harap-harapan: Tungo sa isang Filipinong “KOINE”. Quezon City: KWF.

Casanova, A. P., Batua, L. P., et al. (1984). Sining ng komunikasyon (Pandalubhasaan). Quezon City: Rex Printing Company.

De la Serna, L. L. (1994). Exploring the Cebuano Language. Cagayan de Oro City: Solo Printing and Graphic Design.

Fruto, J. O. (1969). English lexical erros induced by Cebuano grammatical ang lexical pattern. Cebu City: University of San Carlos.

Guamen, P. C., at Espiritu, C. C. (1995). Tanging gamit ng Filipino. Quezon City: Rex Book Store.

Lachica, V. S. (1994). Komunikasyon at linggwistika. Manila: Rex Book Store.

Mante, R. F. (1971). The educational signifance of the difficulties of Cebuanospeakers in learning English: An attempts at linguistic cultural explanation. Cebu City: University of San Carlos.

Martelino, J. I. (2016). A study of affixes and morphophonemic changes in nouns Maranao. Manila: Philippine Normal University.

Matic, A. J. (1996). Lingguistics. USA: Oxford University Press.

Moksir, M. (1970). Hambingan pag-aaralng mga panlapi sa Tagalog at Maranaw. Surian ng Wikang Pambansa.

Nopuli, D. J. (1996). Lingguistics. USA. Oxford University Press.

Ongoco, C. T. (1990). Mga tulong sa pag-aaral sa Noli Me Tangere. Quezon City: Manlapaz Publishing Company.

Oreiro, A. (2016). A study of affixes and morphophonemic changes in Itawit noun and adjectives (Masteral thesis). Philippine Normal University, Manila, Philippines.

Rubin, L. T. (1992). Wika sa lipunang Pilipino. Quezon City: Rex Book Store.

Santiago, A. O. (1977). Panimulang linggwistika sa Pilipino. Manila: Rex Book Store.

Tan, E. A. (1967). A morpho-syntactic analysis of the Cebuano-Bisaya language and it's corresponding influence on the learning of Pilipino by Cebuano speakers. Cebu City: University of San Carlos.

Tanangkingsing, M. M. (2009). A functional grammar of Cebuano. Cebu City: University of San Carlos.

Tiukinhoy, N. O. (1981). Isang pahambing na pagsusuri ng mga pandiwang di-karaniwan sa wikang Surigaonon at sa wikang Filipino sa larangan ng morpolohiya ayon sa taltong aspekto ng mga pandiwa. Cebu City: Cebu State College.

Trosdal, M. B. (1992). Grammar of the Cebuano language. Cebu City: Salvador \& Pilar Sala Foundation.

Uy, M. L. (1971). Mga pagkakaugnat ng mga tunog at baybay ng mga "Cognate” sa Pilipino at Sebuano-Bisaya”. Cebu City: Dalubhasaang Normal sa Pilipinas.

Wolf, J. U. (1966). Beginning Cebuano Part I. New Haven \& London: Yale University Press. 
Bacalla, L. 